\title{
Successful management of severe lupus crescentic nephritis and pneumonitis with weekly cyclophosphamide and plasmapheresis
}

\begin{abstract}
Systemic lupus erythematosus (SLE) may present with a variety of pulmonary manifestations. The most common is infection however lupus pneumonitis can be presented with a picture like infectious pneumonia. Lupus pneumonitis is a high suspicious in a postpartum young female presenting with pulmonary infiltrates. Type II rapidly progressive glomerulonephritis (RPGN) caused by immune complexes deposition accounts for $25 \%$ of RPGN. In-here, we present a catastrophic lupus pneumonitis and RPGN who responded well to intensive protocol of weekly cyclophosphamide and plasmapheresis.
\end{abstract}

Keywords: lupus pneumonitis, lupus erythematosus, cyclophosphamide, plasmapheresis, pulmonary infiltrates, antibodies
Volume 8 Issue 5 - 2020

\author{
Ahmed Akl, ${ }^{1,7}$ Nahid Janodi, ${ }^{2}$ Souha Issa, ${ }^{3}$ \\ Nabih Hammad, ${ }^{4}$ Wail Tashkandi, ${ }^{4}$ Sayed \\ Asghar, ${ }^{5}$ Hassan A Shabana, ${ }^{5,8}$ Nezar Bahabri, ${ }^{6}$ \\ Hanadi Alhozali, ${ }^{9}$ Mohamed Azzam ${ }^{4}$ \\ 'Nephrology section, Internal Medicine Department, DR. \\ Soliman Fakeeh Hospital, Saudi Arabia \\ ${ }^{2}$ Rheumatology section, Internal medicine department, DR. \\ Soliman Fakeeh Hospital, Saudi Arabia \\ ${ }^{3}$ Gastroenterology section, Internal medicine department, DR. \\ Soliman Fakeeh Hospital, Saudi Arabia \\ ${ }^{4}$ Intensive care department, DR. Soliman Fakeeh Hospital, Saudi \\ Arabia \\ ${ }^{5}$ Chest \& respiratory diseases medicine, DR. Soliman Fakeeh \\ Hospital, Saudi Arabia \\ 'Infectious diseases section, Internal medicine department, DR. \\ Soliman Fakeeh Hospital, Saudi Arabia \\ ־Urology \& Nephrology Center, Mansoura University, Egypt \\ ${ }^{8}$ Chest diseases Department, Faculty of medicine, Cairo \\ University, Egypt \\ 'Internal Medicine Department, King Abdulaziz University, Saudi \\ Arabia
}

Correspondence: Ahmed AkI MD, FACP, FASN, DR. Soliman Fakeeh hospital, Jeddah, KSA, Urology \& Nephrology center, Mansoura University, Egypt, Email aikl200।@yahoo.com

Received: August 21, 2020 | Published: September 2I, 2020
Abbreviations: SLE, systemic lupus erythematosus; RPGN, rapidly progressive glomerulonephritis; BAL, broncho-alveolar lavage; OP, organizing pneumonia; $\mathrm{CTD}$, connective tissue disease

\section{Introduction}

Systemic Lupus erythematosus (SLE) is identified by production of antibodies against various cellular antigens derived from nucleus, cytoplasm and cell membrane. Twenty five percent of rapidly progressive glomerulonephritis (RPGN) is secondary to immune complex deposition and is classified as type II. RPGN can be a presentation of SLE, acute proliferative glomerulonephritis, HenochSchönlein purpura and IgA nephropathy. ${ }^{1}$ Pulmonary signs of SLE may present with a various spectrum of diseases such as pleuritis, pneumonia, pulmonary embolism, pneumothorax and or pulmonary hemorrhage. ${ }^{2-4}$ Immune complex deposition injury are the main player in pulmonary complications. As well, infection contribute in most common forms of pulmonary involvement. ${ }^{4}$ Lupus acute pneumonitis picture overlaps infectious acute pneumonia the incidence ranges from 0.9-11.7 \%. ${ }^{3}$ Lupus acute pneumonitis present with dry cough, fever, dyspnea, and occasional hemoptysis. ${ }^{5}$ Suspected cases of lupus pneumonitis usually post-partum young female with unexplained pulmonary infiltrates. ${ }^{6}$ Blood and sputum cultures are essential to rollout infective causes. In overlapping cases bronchoscopy or open-lung biopsy is necessary to set the proper diagnosis of other conditions, such as pneumonia, alveolar hemorrhage, and other acute pulmonary processes that resemble lupus pneumonitis clinically and radio graphically. $^{7}$

\section{Case report}

24-year-old primigravida female, SLE was diagnosed 4 years back at her hometown in Pakistan. Diagnosis was based on clinical criteria including oral ulcer, photosensitivity (malar rash) and positive serology for anti-neutrophilic antibodies (ANA), anti-DS DNA, antiSM positive and low C3, C4. Recently moved to Saudi Arabia, on her first visit to our hospital she was pregnant in the first month. On presentation she had active lupus with alopecia, no joint pain, no joint swelling, no family history of rheumatological disease, no oral ulcers. Her serum creatinine was $0.6 \mathrm{mg} / \mathrm{dl}$, active urinary sediment (heavy proteinuria, microscopic hematuria), 24-hour urinary protein was $5 \mathrm{gm} /$ day, Hb13.4 gm/1, ESR 8, PLT 268, positive ANA, positive anti-DS DNA, intermediate positive anticardiolipin antibodies, low C3, normal C4, Anti SSA/SSAB (Anti-RO/Anti-LA) negative. Renal ultrasound showed Bilateral grade I medical nephropathy, with preserved renal size and parenchymal thickness, no renal calculi or hydronephrosis and no perinephric collection. She was maintained on tacrolimus $1 \mathrm{mg}$ every 12 hours, prednisolone $2.5 \mathrm{mg}$ OD and plaquenil $400 \mathrm{mg}$ per day. She was on cellcept $1000 \mathrm{mg}$ per day, 
stopped one month ago because of pregnancy and it was not replaced with azathioprine. In view of her clinical and laboratory results she was advised to have renal biopsy.

Patient lost follow up for 3 weeks then she was presented with miscarriage to the obstetric department and was subjected to dilatation \& curettage $(\mathrm{D} \& \mathrm{C})$ with rapidly progressive renal failure with creatinine $2.4 \mathrm{mg} / \mathrm{dl}$, moderate edema lower limb and dry cough. After clearance from infection, pulse methylprednisolone $1 \mathrm{gm} /$ day was initiated, furosemide $40 \mathrm{mg}$ every 8 hours with no response that later was converted to furosemide infusion $10 \mathrm{mg} /$ hour and Albumin $50 \mathrm{ml} \mathrm{20 \%}$ every 12 hours to improve her volume overload status and chest status. Echocardiography revealed rheumatic heart in the form of mitral valve regurge, tricuspid valve regurge, pulmonary hypertension, congestive heart failure with BNP 14000. Chest x-ray showed bilateral mid- zone and basal veiling, likely spreading effusion with underlying consolidation, accentuated bronchovascular markings, Blunted both costophrenic angles Figure 1 (A). On day 2 post pulse methylprednisolone the patient developed shortness of breath, worsening of her oxygen level, her chest x-ray showed extensive pulmonary oedema, basal consolidation. High resolution CT chest showed bilateral mid-zonal and lower lobe ground glass opacities, bilateral pleural effusions, mild pericardial effusion Figure 1 (B). Patient developed sudden attack of tachypnea, shortness of breath, hypoxemia that necessitate urgent endotracheal intubation and mechanical ventilation. On view of worsening of her clinical condition and chest $\mathrm{x}$-ray with cessation of urine output and rise of serum creatinine $6 \mathrm{mg} / \mathrm{dl}$, cyclophosphamide $500 \mathrm{mg}$ IV was initiated after clearance from infectious disease department.
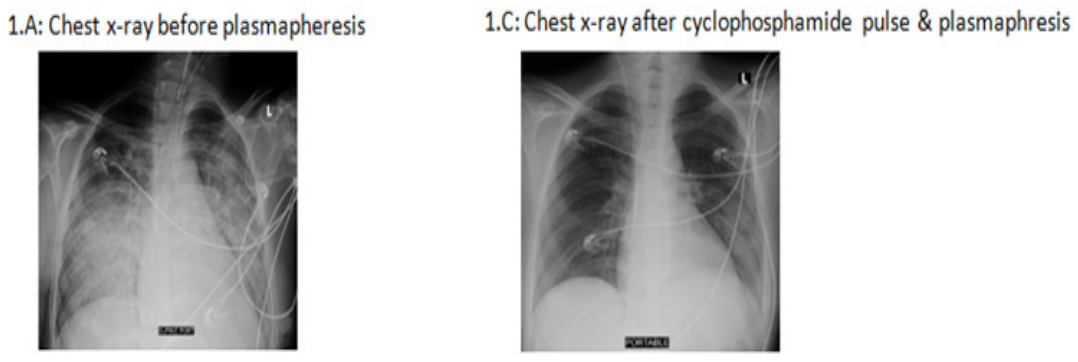

1.B: High resolution ct chest

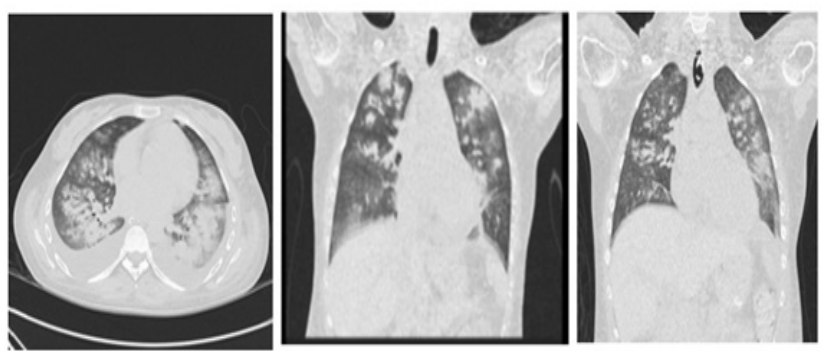

Figure I Chest radiography: I (A) Chest x-ray before start of plasmapheresis shows extensive pulmonary edema, basal consolidation. I(B) High resolution CT showed bilateral mid-zonal and lower lobe ground glass opacities, bilateral pleural effusions, mild pericardial effusion. I(C) Chet x-ray shows recovery after pulse cyclophosphamide and plasmapheresis.

After one week, there was no improvement of renal function as patient was maintained on alternate-day ultra-filtration and hemodialysis sessions, urine output was less than $0.5 \mathrm{ml} / \mathrm{kg} / \mathrm{hr}$. Renal biopsy was done and analyzed at Express Med laboratories, ElBahrain. Light microscopy examination of glomeruli revealed variable segmental endocapillary hypercellularity, crescents with $30 \%$ cellular, $25 \%$ fibrous and the rest glomeruli had fibro-cellular crescents. Occasional wire-loops were noted. Basement membranes were focally \& segmentally split. Tubules display moderate acute injury and focally contain red cell and hyaline casts. Chronic tubulointerstitial damage is less than $5 \%$. Occasional mononuclear cell infiltrates are seen in the interstitium but no active tubulitis. Eosinophils were not seen. one of the arterioles showed an organizing thrombus. Electron microscopy revealed enlarged podocytes microvillation, cytoplasmic vacuolation and with $30 \%$ foot process effacement along capillary surface. Electron dense deposits were seen predominantly in subendothelial and mesangial areas with several large and small ones in subepithelial locations, some of these transmembranous. Some subendothelial deposits have a vague internal fibrillary structure. There is no significant mesangial cell interposition. Tubulo-reticular inclusions were not seen (Figure 2).
Chest condition failed to improve, a second pulse of cyclophosphamide was given followed by partial improvement of chest condition regarding ventilation parameters and radiology (x-ray). High resolution CT (HRCT) chest was requested, during transferring the patient to $\mathrm{CT}$ room, she developed sudden attack of hemoptysis associated with sudden worsening of her chest $\mathrm{x}$-ray. Urgent bronchoscopy was done and revealed alveolar hemorrhage. Plasmaphereses was initiated and combined with ultrafiltration. After 3 sessions of plasmapheresis marvelous clinical recovery was noted, patient urine output started to improve with satisfactory levels, her creatinine dropped to $1.6 \mathrm{mg} / \mathrm{dl}$ without hemodialysis. Her chest $\mathrm{x}$-ray improved, patient oxygenation and ventilation parameters improved and successfully weaned from mechanical ventilation. Patient was transferred to the ward, after 5 days from the last cyclophosphamide pulse she developed sudden grand male fits, magnetic reasoning image brain (MRI) revealed picture coop with lupus cerebritis (Figure 3). The $3^{\text {rd }}$ dose of cyclophosphamide was given and she was discharged home with improved condition to be maintained on mycophenolate mofetil $500 \mathrm{mg}$ every 12 hours plus $40 \mathrm{mg}$ prednisolone oral with plan of weaning in the following months according to her general condition. 

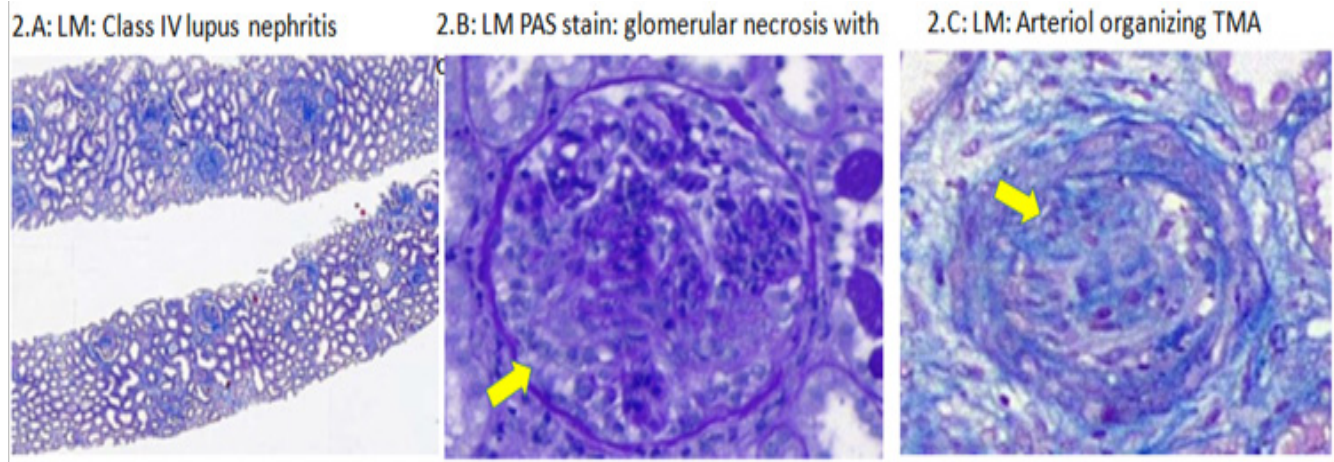

\section{D: EM: Subendothelial \& Mesangial deposites}

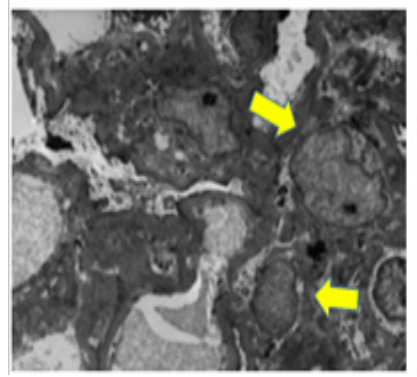

Figure 2 Renal biopsy histopathology: 2(A) LM: Class IV lupus nephritis. 2(B) LM PAS stain: glomerular necrosis with crescent. 2(C) LM: organizing TMA. 2(D) EM: subendothelial \& mesangial deposits.

\section{Discussion}

Lupus pneumonitis is a life-threatening condition associated with flare-up of SLE. The radiological manifestations of lupus pneumonitis rarely include cavitation and usually there is either single or multiple consolidations at the basal and bilateral lung zones with bilateral pleural effusion and pulmonary arterial hypertension. ${ }^{8}$ While Lupus pneumonitis respond to steroids and cyclophosphamide, mortality is high reaching $50 \%$ of cases. ${ }^{9}$ The diagnosis of Lupus pneumonitis is difficult and relay mainly on rolling out other etiologies of lung infiltration. In our case infective pneumonia was excluded by repeated sputum culture and single Broncho-Alveolar Lavage (BAL) fluid analysis and culture. The alveolar hemorrhage was excluded by the absence of hemoptysis and BAL analysis for Hemosiderinladen macrophage. The syndrome of acute reversible hypoxemia was excluded by the presence of abnormal chest X-ray and HRCT scan of thorax. Organizing pneumonia (OP), a rare complication of rheumatoid arthritis that may simulate Lupus pneumonitis, usually affect elderly with average age of 50-60 years. OP is subacute in nature begins with flu-like symptoms with no fever and associated with cough, malaise, mild dyspnea, anorexia and weight loss. ${ }^{10}$ In connective tissue disease (CTD) associated OP has radiological characteristics of reticulo-nodular pattern while idiopathic pulmonary fibrosis is usually patchy alveolar infiltration. ${ }^{11}$ Prolonged treatment is needed in CTD associated OP and relapse is common upon reduction of corticosteroids to less than $20 \mathrm{mg} /$ day. ${ }^{10} \mathrm{In}$ our case lupus pneumonitis diagnosis was established by the typical clinical manifestations, HRCT findings and dramatic response to corticosteroids. Wan SA et al. in their retrospective study, all patients presented with fever, cough and pulmonary infiltrates on chest radiographs. HRCT which was performed in two patients, showed bilateral patchy consolidations with ground glass opacities. While all patient received steroids, cyclophosphamide and immunoglobulins, the mortality was $40 \%{ }^{12}$

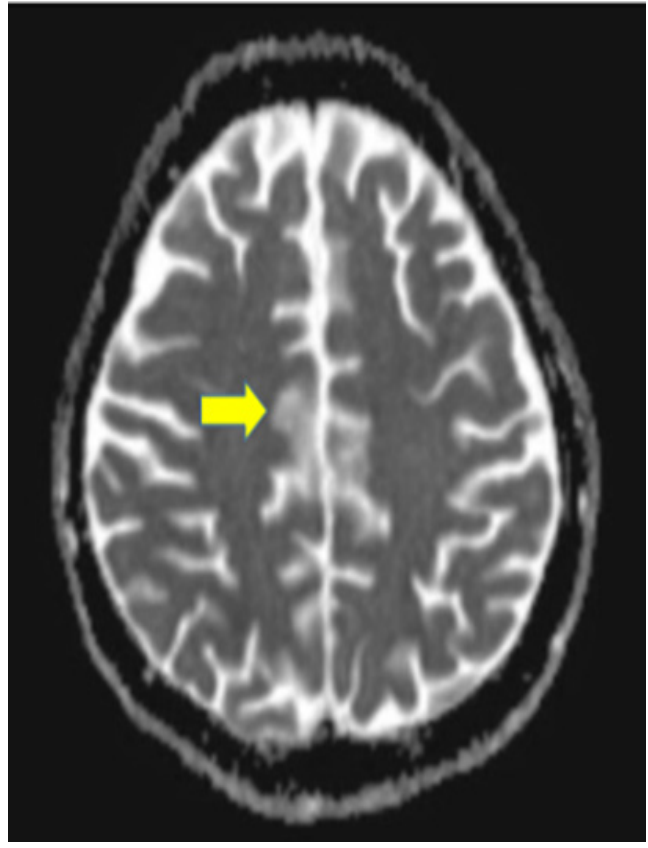

Figure 3 Brain MRI: picture coop with lupus cerebritis.

\section{Conclusion}

In conclusion, catastrophic lupus presentation with RPGN and lupus pneumonitis needs efficient diagnosis and careful management.

\section{Acknowledgments}

None. 


\section{Conflicts of interest}

The author declares there is no conflict of interest.

\section{References}

1. Kaori Mochizuki, Ken Kayakabe, Keiju Hiromura, et al. Successful treatment of severe crescentic lupus nephritis by multi-target therapy using tacrolimus and mycophenolate mofetil. CEN Case Rep. 2015;4(2):126130.

2. Beresford MW, Cleary AG, Sills JA, et al. Cardio-pulmonary involvemen in juvenile systemic lupus ery-thematosus. Lupus. 2005;14(2):152-158.

3. Ciftçi E, Yalçinkaya F, Ince E, et al. Pulmonary involvement in childhoodonset systemic lupus erythematosus: a report of five cases. Rheumatology. 2004;43(5):587-591.

4. Murin S, Wiedemann HP, Matthay RA. Pulmonary manifestations of systemic lupus erythema-tosus. Clin Chest Med. 1998;19(4):641-665.

5. Matthay RA, Schwarz MI, Petty TL, et al. Pulmonary manifestations of systemic lupus erythematosus: review of twelve cases of acute lupus pneumonitis. Medicine (Baltimore). 1975;54(5):397-409.
6. Trager J, Ward MM. Mortality and causes of death in systemic lupus erythematosus. Curr Opin Rheumatol. 2001;13(5):345-351.

7. Haupt HM, Moore GW, Hutchins GM. The lung in systemic lupus erythematosus. Analysis ofthe pathologic changes in 120 patients. Am J Med. 1981;71(5):791-798.

8. Matthay RA, Schwarz MI, Petty TL, et al. Pulmonary manifestations of systemic lupus erythematosus: Review of twelve cases of acute lupus pneumonitis. Medicine. 1975;54:397-409.

9. Wiedemann HP, Matthay RA. Pulmonary manifestations of systemic lupus erythematosus. J Thorac Imaging. 1992;7:1-18.

10. Cordier JF. Cryptogenic organising pneumonia. Eur Respir J. 2006; 28:422-446.

11. Lamblin C, Bergoin C, Saelens $\mathrm{T}$, et al. Interstitial lung diseases in collagen vascular diseases. Eur Respir J. 2001;18:69S-S80.

12. Wan SA, Teh CL, Jobli AT. Lupus pneumonitis as the initial presentation of systemic lupus erythematosus: case series from a single institution. Lupus. 2016;25(13):1485-1490. 